Research in Astron. Astrophys. 0000 Vol. 0 No. 00, 000-000

http://www.raa-journal.org http://www.iop.org/journals/raa

$\boldsymbol{R}$ esearch in

Astronomy and

Astrophysics

\title{
On Pulsar-Driven Mass Ejection in Low-Mass X-ray Binaries
}

\author{
Lei Fu and Xiang-Dong Li \\ Department of Astronomy, Nanjing University, Nanjing 210093, China; fl19821110@yahoo.com.cn; \\ lixd@nju.edu.cn \\ Key Laboratory of Modern Astronomy and Astrophysics, Ministry of Education, Nanjing 210093, \\ China \\ Received [year] [month] [day]; accepted [year] [month] [day]
}

\begin{abstract}
There is accumulating evidence for mass ejection in low-mass X-ray binaries (LMXBs) driven by radio pulsar activity during X-ray quiescence. In this paper we consider the condition for mass ejection by comparing the radiation pressure from a millisecond pulsar, and the gas pressure at the inner Lagrange point or at the surrounding accretion disk. We calculate the critical spin period of the pulsar below which mass ejection is allowed. Combining with the evolution of the mass transfer rate, we present constraints on the orbital periods of the systems. We show that mass ejection could happen in both wide and compact LMXBs. It may be caused by transient accretion due to thermal instability in the accretion disks in the former, and irradiation-driven mass-transfer cycles in the latter.
\end{abstract}

Key words: pulsars: general-binaries: general

\section{INTRODUCTION}

Currently there are 144 millisecond pulsars (MSPs) (pulse periods $p<10 \mathrm{~ms}$ ) in the ATNF pulsar database, 86 of which are located in binary systems. MSPs are traditionally considered as the descendants of old neutron stars which were spun up by accretion from their companions in low-mass X-ray binaries (LMXBs) (Bhattacharya \& van den Heuvel 1991 for a review). Typically, accretion of $\Delta M \sim 0.1 M_{\odot}$ mass is sufficient to accelerate a slowly rotating neutron star to milliseconds (Burderi et al. 1999).

More recent investigations demonstrate that perhaps most of the current LMXBs have evolved from systems with intermediate-mass $\left(\gtrless 1.5 M_{\odot}\right)$ donor stars, i.e., intermediate-mass X-ray binaries (IMXBs) (Davies \& Hansen 1998; King \& Ritter 1999; Podsiadlowski et al. 2002; Pfahl et al. 2003). Because the donor star is initially more massive than the neutron star, mass transfer proceeds rapidly on a timescale $\lesssim 10^{6}$ years. Little mass is accreted during this phase since the mass transfer rate is usually much higher than the Eddington limit for the neutron star, until the donor mass becomes comparable with the neutron star mass and the binary evolves to be an LMXB (Tauris \& Savonije 1999; Podsiadlowski et al. 2002). It is believed that in this later phase the neutron star accretes sufficient mass and experiences the recycling process.

There is evidence that mass transfer in the LMXB phase is also highly nonconservative. The companion star of MSPs is usually a white dwarf of mass $\sim 0.1-0.4 M_{\odot}$, the progenitor of which is expected to be a star with initial mass $\gtrsim 1 M_{\odot}$ (Tauris \& Savonije 1999; Pfahl et al. 2003). So the lost mass from the companion is about $0.6-0.9 M_{\odot}$. If the mass transfer is conservative, the expected minimum mass of the MSPs would exceed $2 M_{\odot}$. However, measurements of the pulsar masses suggest that only part of the transferred mass was accreted by the neutron stars (Thorsett \& Chakrabarty 1999; 
Zhang et al. 2011: Kizilatan, Kottas, \& Thorsett 2010. See also Table 1 for a list of binary MSPs with measured masses), except that in a few case the masses of MSPs are as high as $\sim 2 M_{\odot}$ (e.g., PSRs B1957+20, van Kerkwijk \& Breton 2011; J0751+1807, Nice et al. 2005; J1614-2230, Demorest et al. 201017). So in most of the systems, the mass transfer must have been nonconservative.

The mechanism of mass ejection in X-ray binaries was first introduced by Illarionov \& Sunyaev(1975) as the "propeller" effect, i.e., the accretion flow is centrifugally prohibited at the magnetosphere of the neutron star, at the cost of the slow down of star's spin. As pointed out by Burderi et al. (2001), the efficiency of the propeller effect is at most $\sim 50 \%$, and the pulsars should still be more massive than observed. It was later suggested that, if the mass transfer rate varies by large amplitude, the neutron star may become an MSP when the accretion rate is very low, and the energetic pulsar wind may disrupt the accretion disk around the neutron star, so that the transferred mass will escape from the binary across the inner Lagrangian point $L_{1}$, which we call pulsar-driven mass ejection (Burderi et al. 2001; Burderi, D'Antona \& Burgay 2002). Since this process occurs at $L_{1}$, at which the binding energe is very small, the efficiency of ejeciton may reach unity. An alternative mechanism is that the energetic wind or hard X-ray radiation from the MSP may evaporate it's low-mass companion (van den Heuvel \& van Paradijs 1988; Ruderman, Shaham \& Tavani 1989; Shaham \& Tavani 1990; Shaham \& Tavani 1991; Podsiadlowski 1991).

Direct observational evidence for mass ejection induced by pulsar activity may come from the "black widow pulsars". They are MSPs in binary systems, undergoing very wide eclipse, implying obscuration by intense wind from the secondary (Fruchter, Stinebring, \& Taylor 1988). Another interesting example is PSR J1740-5340, an eclipsing MSP with a spin period of $3.65 \mathrm{~ms}$ and orbital period of 32.5 hr, located in the globular cluster NGC 6397 (D'Amico et al. 2001). Long lasting and sometimes irregular radio eclipses, and the shape of the optical light curve demonstrated the presence of matter around the system (Ferraro et al. 2001), suggesting that PSR J1740-5340 is an example of a system in the pulsar-induced ejection phase.

In this paper we investigated the conditions for mass ejection in LMXBs caused by the turn-on of pulsar activity. The basic idea is similar to that in Burderi et al. (2001). However, we consider direct mass ejection at both $L_{1}$ and $L_{2}$, which requires that the pulsar's radiation pressure is strong enough to drive material out of these points, and was ignored in Burderi et al. (2001). For mass ejection due to disruption of the accretion disks by the pulsar's pressure, we adopt a more realistic accretion disk model to evaluate the disk pressure. In section 2 we calculated the critical periods $p_{\text {cr }}$ at which the pulsar wind pressure $P_{\mathrm{psr}}$ equals the donor's gas pressure $P_{\mathrm{L} 1}$ at the $L_{1}$ point, and $P_{\mathrm{disk}}$ in the accretion disk respectively. The condition for outflow from $L_{2}$ is also considered. In section 3 we put possible constraints on the orbital periods by combining the evolution of LMXBs. We briefly discuss the implications of our results in section 4 .

\section{THE CRITICAL PERIODS}

\subsection{Mass Flow Through the Inner Lagrange Point $L_{1}$}

We consider a semi-detached binary containing a neutron star and a donor star that fills it's Roche lobe. The atmospheric material is overflowing through $L_{1}$ towards the neutron star. At $L_{1}$ the gas flow is confined in a "nozzle" with radius $H$ and moves with a speed close to the sound velocity (Frank, King, \& Raine 2002). The mass transfer rate is expressed as

$$
\dot{M} \simeq \pi H^{2} c_{s}\left(L_{1}\right) \rho\left(L_{1}\right),
$$

where $c_{s}\left(L_{1}\right)$ and $\rho\left(L_{1}\right)$ are the local sound velocity and the gas density at the $L_{1}$ point. To estimate the magnitude of $H$, we consider the balance between the Roche potential and the mean kinetic energy of

\footnotetext{
${ }^{1}$ In a recent paper, Tauris et al. (2011) suggested that PSR J1614-2230 must have been born with a mass significantly exceeds $1.4 M_{\odot}$.
} 
the material, and find that matter escapes in a patch of radius (see also Frank, King, \& Raine 2002; Li et al. 2010)

$$
H \simeq \frac{c_{s}\left(L_{1}\right)}{\sqrt{f_{1}} \omega}
$$

where

$$
f_{1}(q)=\frac{1}{2}\left[\frac{1}{(1+q)\left(0.5-0.227 \log _{10} q\right)^{3}}+\frac{q}{(1+q)\left(0.5+0.227 \log _{10} q\right)^{3}}-1\right]
$$

where $q$ is the mass ratio of the donor and the neutron star, ranging from $\sim 0.1$ to $\sim 3$ for I/LMXBs. The mass transfer rate can then be written as

$$
\dot{M}=1.03 \times 10^{6} P_{\mathrm{orb}, \mathrm{hr}}^{2} c_{s}^{3}\left(L_{1}\right) \rho\left(L_{1}\right) f_{1}^{-1}(q) \mathrm{g} \mathrm{s}^{-1},
$$

where $P_{\mathrm{orb}, \mathrm{hr}}$ is the orbit period of the system in units of hour. Consequently, the gas pressure at $L_{1}$ is

$$
P_{L 1}=\rho\left(L_{1}\right) c_{s}^{2}\left(L_{1}\right)=6.15 \times 10^{3} \dot{M}_{-10} P_{\text {orb }, \mathrm{hr}}^{-2} c_{s 6}^{-1} f_{1}{\mathrm{dyn} \mathrm{cm}^{-2}}^{-2}
$$

where $\dot{M}_{-10}=\dot{M} / 10^{-10} M_{\odot} \mathrm{yr}^{-1}$, and $c_{s 6}=c_{s} / 10^{6} \mathrm{cms}^{-1}$.

\subsection{Equilibrium at $\boldsymbol{L}_{1}$ and $\boldsymbol{L}_{2}$}

Once the material from the donor star is captured by the neutron star at the circularization radius, an accretion disk will form due to dissipative processes and internal torque in it. Sufficiently long time accretion on to the neutron star can spin it up to millisecond periods. If the accretion rate drops due to some reasons, the inner radius of the accretion disk may move outside of the light cylinder (with radius $R_{L C}$ ) of the neutron star, and the neutron star changes from an accreting $\mathrm{X}$-ray source to be a rotation-powered pulsar, emitting out radio, optical, X-ray, $\gamma$-ray photons and high energy particles. If this process is dominated by the magnetic dipole radiation, the pulsar wind pressure at $L_{1}$ can be expressed as

$$
P_{\mathrm{psr}}\left(L_{1}\right)=8.23 \times 10^{2} B_{s 8}^{2} P_{\mathrm{orb}, \mathrm{hr}}^{-4 / 3} p_{\mathrm{ms}}^{-4} R_{n 6}^{6} m_{1}^{-2 / 3} f_{2}^{-2}(q) \mathrm{dyn} \mathrm{cm}^{-2},
$$

where $m_{1}$ and $R_{n 6}$ are the mass and radius of the pulsar in units of solar mass and $10^{6} \mathrm{~cm}$ respectively, $B_{s 8}$ the surface magnetic field in units of $10^{8} \mathrm{G}, p_{\mathrm{ms}}$ the pulsar period in units of millisecond, and

$$
f_{2}(q)=(1+q)^{1 / 3}\left(0.5-0.227 \log _{10} q\right) .
$$

When the pulsar wind pressure is larger than the gas pressure $\left(P_{L 1}\right)$ at $L_{1}$, the accretion process is prohibited, and mass ejection will happen. Combining Eqs. (5) and (6) we can get a critical period at which material flow through $L_{1}$ will be ejected,

$$
p_{\text {cr }} \simeq(0.61 \mathrm{~ms}) f_{1}^{-1 / 4} f_{2}^{-1 / 2} \dot{M}_{-10}^{-1 / 4} c_{s 6}^{1 / 4} B_{s 8}^{1 / 2} P_{\mathrm{orb}, \mathrm{hr}}^{1 / 6} R_{n 6}^{3 / 2} m^{-1 / 6} \text {. }
$$

When the secondary star evolves to be of very low mass, it may be the outer Lagrangian point $L_{2}$ rather $L_{1}$ with the lowest potential, due to the pulsar's radiation pressure force on the Roche potential of the secondary. In this case, Roche-lobe overflow will not occur towards the pulsar, but into a circumbinary disk/outflow (as is indeed observed for PSR 1957+20). According to Phillips \& Podsiadlowski (2002), the condition for outflow from $L_{2}$ can be defined as $\delta_{\max } \equiv q L / L_{E d d}$ becomes larger than a critical value $\delta_{\text {crit }}$ (where $L$ and $L_{E d d}$ are the irradiating pulsar's luminosity and the Eddington luminosity of the secondary, respectively), or equivalently

$$
\frac{L \kappa}{4 \pi c G M}=\delta_{c r i t}
$$


where

$$
L=\left(\frac{2 R_{n}^{6}}{3 c^{3}}\right) B_{s}^{2}\left(\frac{2 \pi}{p}\right)^{4}
$$

and $\kappa$ is the mean photospheric opacity. The resulting critical spin period is

$$
p_{\mathrm{cr}, \mathrm{L} 2}=2 \pi\left(\frac{\kappa B^{2}}{4 \pi c G M \delta_{\text {crit }}} \frac{2 R_{n}^{6}}{3 c^{3}}\right)^{1 / 4}
$$

or

$$
p_{\mathrm{cr}, \mathrm{L} 2} \simeq(0.48 \mathrm{~ms}) \kappa_{0.4}^{1 / 4} m^{-1 / 4} B_{s 8}^{1 / 2} R_{n 6}^{3 / 2}
$$

with $\delta_{\text {crit }}=0.0564$ when $q=0.1$ (Phillips \& Podsiadlowski 2002). Here $\kappa_{0.4}=\kappa / 0.4 \mathrm{~cm}^{2} \mathrm{~g}^{-1}$ may be much larger than unity for the photosphere of very low-mass stars, so Eq. (12) presents a lower limit of the critical period. Obviously, depending on the mass ratio, this criterion may be more relevant for very low-mass binaries.

\subsection{Equilibrium with the disc}

Now we consider possible disruption of the accretion disk by the pulsar's pressure. The standard geometrically thin $\alpha$-disk model was established by Shakura \& Sunyaev (1973). However, there is observational evidence showing deviation from the theoretical predictions - in cataclysmic variables (CVs) and LMXBs the outer disk edge seems to be thick and structured (Shafter \& Misselt 2006; Hakala et al. 1999). Begelman \& Pringle (2007) argued that, the magnetorotational instability (MRI), which successfully accounts for angular momentum transport in accretion disks (Balbus \& Hawley 1998), can amplify the toroidal magnetic field to a point at which magnetic pressure far exceeds the combined gas and radiation pressure in the disk. This additional pressure support makes the disk thicker than in the $\alpha$ disk model, in accordance with observations. In this magnetic-dominated disk model, the Alfvén speed associated with the toroidal field is roughly the geometric mean between the Keplerian speed and the sound speed. Thus the magnetic pressure is $P_{B} \simeq \rho c_{g} v_{k}$. The total disc pressure can be derived to be (see appendix)

$$
P_{\text {disk }} \simeq P_{B}=1.02 \times 10^{13} \alpha^{-17 / 18} m^{61 / 36} \dot{M}_{-10}^{8 / 9} R_{6}^{-91 / 36} R_{n 6}^{-1}\left[1-\left(\frac{R_{n 6}}{R_{6}}\right)^{1 / 2}\right]^{-1 / 9} \mathrm{dyn} \mathrm{cm}^{-2},
$$

where $R_{6}$ is the disk radius in unit of $10^{6} \mathrm{~cm}$. As pointed out by Burderi et al. (2001), if the spun-up pulsar is switched on, the accretion disk may be truncated at a radius $R_{\text {stop }}$ due to the energetic pulsar wind. Once $R_{\text {stop }}$ is smaller than the outer radius $R_{\text {out }}$ of the accretion disk, the overflowed material through $L_{1}$ will be ejected, i.e., the system will enter the mass ejection phase.

Traditionally the outer radius of the disk is approximated to be $\sim 70 \%-90 \%$ of the Roche lobe radius $R_{L 1}$ of the primary (Frank, King, \& Raine 2002). Here we take $R_{\text {out }} \simeq 0.8 R_{L 1}$. Combining Eq. (6) and (13), the critical pulsar spin period for mass ejection is

$$
p_{\mathrm{cr}}^{\prime}=(1.94 \mathrm{~ms}) \alpha^{17 / 72} m^{1 / 24} R_{n 6}^{7 / 4} B_{s 8}^{1 / 2} \dot{M}_{-10}^{-2 / 9} P_{\mathrm{orb}, \mathrm{hr}}^{19 / 216} f_{3}
$$

where

$$
f_{3}=(1+q)^{19 / 432}\left[1-0.462\left(\frac{q}{1+q}\right)^{1 / 3}\right]^{91 / 144}\left(0.5-0.227 \log _{10} q\right)^{-1 / 2}
$$




\subsection{Accretion Equilibrium Period}

In the above subsections we show that mass ejection can take place only when the spin period of the neutron star is shorter than either $p_{\mathrm{cr}}$ or $p_{\mathrm{cr}}^{\prime}$. The neutron star's spin is accelerated by both mass accretion and magnetic field-disk interaction during the mass transfer process, until an equilibrium period is reached, at which there is no net torque exerted on the neutron star. According to Ghosh \& Lamb (1979), the spin-up/down rate depends on the fastness parameter $\omega_{\mathrm{s}} \equiv \Omega_{\mathrm{s}} / \Omega_{\mathrm{K}}\left(r_{0}\right)$, i.e., the ratio of the spin angular velocity of the neutron star and the Keplerian velocity of plasma at the inner disk radius $r_{0}$. When $\omega_{\mathrm{s}}$ approaches the critical fastness parameter $\omega_{\mathrm{c}}$, the torque is zero and the spin-up process ceases. Assume $r_{0}=0.5 R_{\mathrm{A}}$, where $R_{\mathrm{A}}$ is the traditional Alfvén radius for spherical accretion

$$
R_{\mathrm{A}}=\left(\frac{B_{s}^{2} R_{n}^{6}}{\dot{M} \sqrt{2 G M}}\right)^{2 / 7}
$$

the equilibrium spin period is

$$
p_{\text {eq }} \simeq(1.95 \mathrm{~ms}) \omega_{0.7}^{-1} R_{n 6}^{18 / 7} B_{s 8}^{6 / 7} m^{-5 / 7} \dot{M}_{-10}^{-3 / 7},
$$

where $\omega_{0.7}=\omega_{\mathrm{c}} / 0.7$. The condition $p_{\mathrm{eq}} \leq p_{\mathrm{cr}}$ or $p_{\mathrm{cr}}^{\prime}$ will constrain the systemic parameters that allow mass ejection to happen in LMXBs.

The neutron star will reach its equilibrium only when the mass transfer rate keeps constant for sufficient long time. When the mass transfer rate changes, the spin period will evolve to new equilibrium on a time scale $t_{\text {spin }} \simeq 2 \pi I \nu_{s} / \dot{M}(G M R)^{1 / 2} \sim 2.3 \times 10^{9} p_{m s}^{-1} M_{-10}^{-1}$, where $I$ is the momentum of inertia of the pulsar. Roughly speaking, if the timescale of the change of the mass transfer rate $t_{\dot{M}}>t_{\text {spin }}$, the spin period of the neutron star is always close to the equilibrium period with current $\dot{M}$. If $\dot{M}$ varies with $t_{\dot{M}} \ll t_{\text {spin }}$, the spin period remains to be close to the previous equilibrium value.

\section{BINARY MSP SYSTEMS THAT FAVOR MATERIAL EJECTION}

\subsection{Mass Transfer Mechanisms}

In this section we investigate the possible influence of the mass transfer rate. As mentioned above, recent studies showed that most LMXBs are likely to originate from IMXBs. During the initial 'IMXB to LMXB' phase, the mass transfer process proceeds on a (sub)thermal timescale, the average mass transfer rate is very high (often super-Eddington for relatively massive companions), so the mass transfer is highly unconservative and the accreted mass by the neutron star is very small. Thus the recycling process should mainly occur in the later LMXB phase. From Eqs. (8), (12) and (14) one can see that mass ejection due to pulsar activity can take place only when the neutron star's spin period is less than a few milliseconds. This is difficult to achieve except for LMXBs and close IMXBs (with case A mass transfer); the outcome of wide IMXB evolution is mildly recycled MSPs with CO WD companions (Tauris 2011). In the former case the LMXB evolution from IMXBs is similar to that of original LMXBs (Podsiadlowski et al. 2002). For these reasons we only consider the evolution of mass transfer rate in LMXBs.

It's well known that mass transfer in LMXBs is driven by nuclear expansion of the donor and orbital angular momentum loss due to magnetic braking and gravitational radiation. By comparing the nuclear evolution time of the donor $t_{\mathrm{nu}}$ and the angular momentum loss timescale $t_{\mathrm{aml}}$, we can divide the evolutionary tracks of LMXBs into the following cases (King, Kolb, \& Burderi 1996).

Case $1 t_{\mathrm{nu}} \ll t_{\mathrm{aml}}$ : In this case the donor has evolved off the main sequence before angular momentum loss shrinks the orbit sufficiently to cause mass transfer. So the donor must be a giant or subgiant, with mass of $\geq 0.8 M_{\odot}$ in order to evolve off main sequence within Hubble time. It's known that the structure of a low-mass giant is determined by the mass of its helium core (Webbink et al. 1983). The mass transfer rate can be expressed by the following relation (King 1988),

$$
\dot{M}_{-10} \simeq 0.21 P_{\mathrm{orb}, \mathrm{hr}}^{0.93} m^{1.47} q^{1.47} .
$$




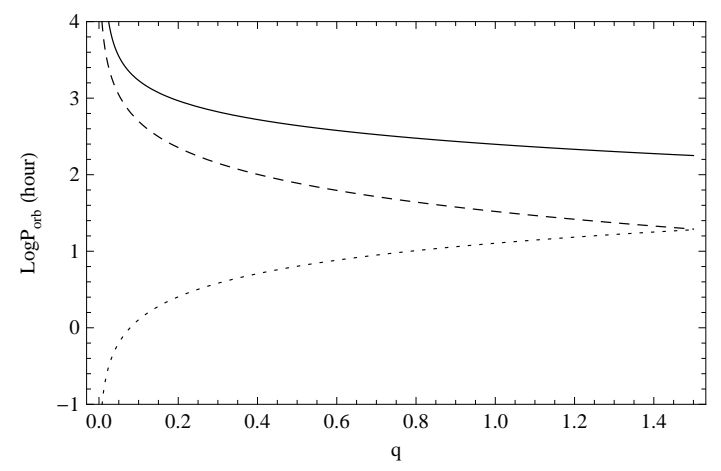

Fig. 1 The relation between $P_{\mathrm{orb}}$,hr and the mass ratio $q$ for LMXBs in case 1 evolution when mass ejection occurs. The solid and dashed lines are for $p_{\mathrm{cr}}=p_{\text {eq }}$, and $p_{\mathrm{cr}}^{\prime}=p_{\text {eq }}$. Above the curves the mass ejection may happen. The dotted line represents the condition when the donor fills its Roche lobe on the main sequence. Here and in the following we adopt $m=1.4$, $R_{n 6}=1, B_{s 8}=5$ and $\alpha=0.1$.

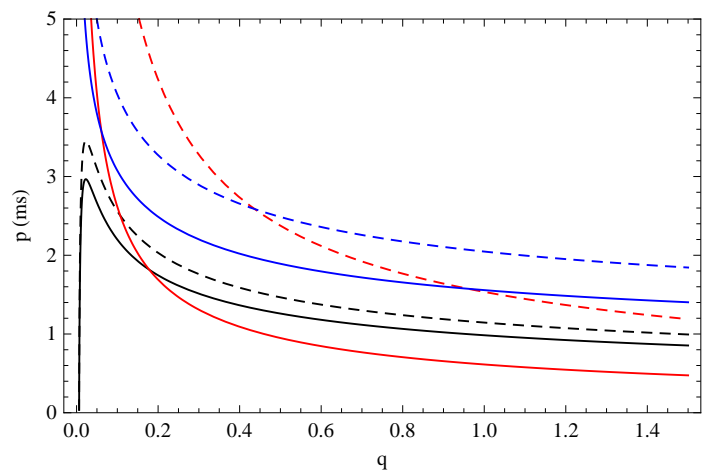

Fig. 2 The relation between critical spin period and the mass ratio $q$ for LMXBs in case 1 evolution. The black, blue and red lines describe $p_{\mathrm{cr}}, p_{\mathrm{cr}}^{\prime}$, and $p_{\text {eq }}$, respectively. The dashed and solid lines are for orbital period $P_{\mathrm{orb}, \mathrm{hr}}=100,1000$, respectively.

Substitute Eq. (16) into Eqs. (8), (11), and (15), when $p_{\mathrm{cr}}=p_{\text {eq }}$ and $p_{\mathrm{cr}}^{\prime}=p_{\text {eq }}$ we obtain the minimum of the orbital period for mass ejection

$$
P_{\text {orb }, \mathrm{hr}}=75.96 \omega_{0.7}^{-2.94} m^{-2.40} q^{-0.78} B_{s 8}^{1.08} f_{1}^{0.76} f_{2}^{1.50} c_{s 6}^{-0.76} R_{n 6}^{3.21},
$$

and

$$
P_{\text {orb }, \mathrm{hr}}^{\prime}=3.22 \omega_{0.7}^{-3.45} \alpha^{-0.83} q^{-1.07} R_{n 6}^{2.83} B_{s 8}^{1.24} m^{-3.78} f_{3}^{-3.45} .
$$

Figure 1 shows the minimum $P_{\mathrm{orb}, \mathrm{hr}}$ and $P_{\mathrm{orb}, \mathrm{hr}}^{\prime}$ against the mass ratio $q$ in the solid and dashed lines, respectively. Note that the minimum orbit period that allows direct mass ejection at $L_{1}$ is $\sim 5$ days, while mass ejection with disk disruption can occur in relatively compact systems. The final product of this kind of evolution is a wide binary with an MSP and a low-mass helium white dwarf.

We also plot $p_{\mathrm{cr}}, p_{\mathrm{cr}}^{\prime}$ and $p_{\text {eq }}$ for constant orbital period with black, blue and red lines in Fig. 2 as a function of mass ratio $q$. Note that in the cases of $P_{\mathrm{orb}, \mathrm{hr}}=100$ (dashed lines) and 1000 (solid lines), $p_{\text {cr }}^{\prime}$ is always larger than $p_{\mathrm{cr}}$.

Case $2 t_{\mathrm{nu}} \sim t_{\mathrm{aml}}$ and $t_{\mathrm{nu}} \gg t_{\mathrm{aml}}$ : In these situations the mass tranfer is driven by angular momentum loss of the system, and

$$
\frac{\dot{M}_{2}}{M_{2}} \sim \frac{\dot{J}}{J}
$$




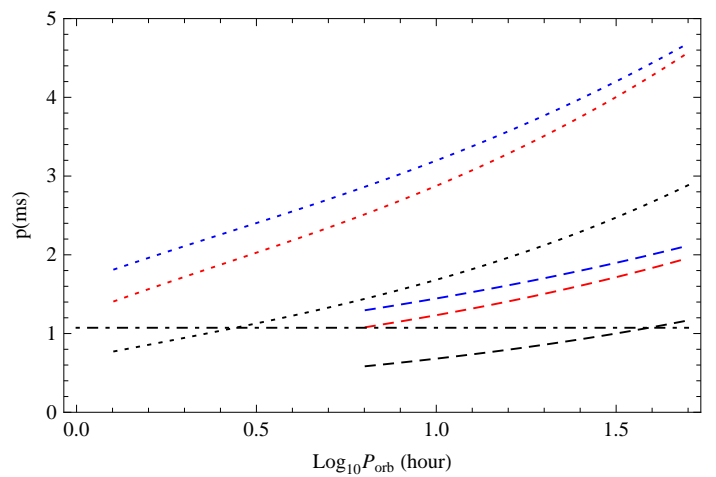

Fig. 3 This figure compares $p_{\mathrm{cr}}, p_{\mathrm{cr}}^{\prime}$, and $p_{\mathrm{eq}}$ as a function of orbital period in case 2 mass transfer. Here $p_{\mathrm{cr}}, p_{\mathrm{cr}}^{\prime}$, and $p_{\mathrm{eq}}$ are plotted in black, blue, and red lines, respectively. The dashed and dotted lines are for $q=0.5$ and 0.1 , respectively. The dot-dashed line represents $p_{\mathrm{cr}, \mathrm{L} 2}$ for $q=0.1$.

where $J$ and $\dot{J}$ are the orbital angular momentum and its derivative, respectively. In the case of $t_{\text {nu }} \sim$ $t_{\mathrm{aml}}$ the donor is mildly evolved when it fills it's Roche lobe, and the radius is slightly bigger than during the main sequence. The orbit period of the system will not change much during the whole evolution. When $t_{\mathrm{nu}} \gg t_{\mathrm{aml}}$, the angular momentum loss of the system is so rapid that mass transfer occurs when the secondary is still on the main sequence, and further nuclear evolution of the secondary is frozen. In this condition the binary orbit will shrink due to the angular momentum loss by magnetic breaking and gravitational radiation. Hence the orbital periods are generally smaller than in case 1 where the secondary is a (sub)giant.

Use the standard forms of gravitational radiation losses (Landau \& Lifschitz 1958) and of magnetic braking (Verbunt \& Zwaan 1981), the mass transfer rate can be written as (King et al. 1996),

$$
\dot{M}_{-10}=552.81 P_{\text {orb }, \mathrm{hr}}^{-2 / 3} q^{7 / 3} m^{5 / 3}+130.65 P_{\text {orb }, \mathrm{hr}}^{-8 / 3} q^{2} m^{8 / 3},
$$

where the first and second terms on the right hand side are for magnetic braking and gravitational radiation, respectively. Figure 3 shows $p_{\mathrm{cr}}, p_{\mathrm{cr}}^{\prime}$, and $p_{\mathrm{eq}}$ as a function of $P_{\mathrm{orb}}$ in black, blue, and red lines, with the mass transfer rate given by Eq. (19). The dashed and dotted lines are for $q=0.5$ and 0.1 respectively. In the case of $q=0.1$ we use Eq. (19) with donor mass $=0.3 M_{\odot}$ to calculate $p_{\text {eq }}$, but remove the first term (i.e., corresponding to magnetic braking) in calculating $p_{\mathrm{cr}}$ and $p_{\mathrm{cr}}^{\prime}$. The reason is that magnetic braking is assumed to vanish abruptly when the secondary mass decreases to $\sim 0.3 M_{\odot}$ (i.e., becomes fully convective), and the subsequent evolution is driven by gravitational radiation only. However, it will take a long time ( $\gtrsim 10^{9} \mathrm{yr}$ ) for the pulsar to reach the new equilibrium period because of the reduced mass transfer rate. So the spin period remains close to the previous one. We have set the limitation on the orbital period $0.11 P_{\mathrm{orb}, \mathrm{hr}} \geq m_{2}$ to guarantee that the secondary fills its Roche lobe before it evolves to be a subgiant. Figure 3 shows that $p_{\text {cr }} \lesssim p_{\text {eq }}<p_{\text {cr }}^{\prime}$, which means that mass ejection via disk disruption might occur. But a firm conclusion is difficult to reach, because of the uncertainties in estimating the critical periods. By the way, outflow from $L_{2}$ seems to require spin periods considerably lower than $p_{\text {eq }}$ for $q=0.1$, disfavoring this possibility for LMXBs. However, this scenario might work in binaries with extreme mass ratio $\left(q \ll 1\right.$, like PSR B1957+20), in which the value of $\delta_{\text {crit }}$ is much smaller (Phillips \& Podsiadlowski 2002).

Note that $p_{\mathrm{eq}} \leq p_{\mathrm{cr}}$ or $p_{\mathrm{cr}}^{\prime}$ is the necessary condition for mass ejection, while switch-on of pulsar emission is critical in expelling the matter overflowing the Roche lobe. This occurs with a temporary reduction of the mass accretion rate, so that the accreting plasma moves out beyond the light cylinder of the neutron star, and the neutron star becomes a generator of magneto-dipole radiation and relativistic particles. The thermal disk instability model (Lasota 2001) is usually invoked to trigger the X-ray outbursts in LMXBs, which may cause the switch-on of the radio pulsar during X-ray quiescence. According to 
Table 1 Properties of binary MSPs with measured masses

\begin{tabular}{cccccr}
\hline PSR Name & $\mathrm{p}(\mathrm{ms})$ & $P_{\text {orb }, \mathrm{hr}}(\mathrm{hr})$ & $M_{p}\left(M_{\odot}\right)$ & $M_{2}\left(M_{\odot}\right)$ & Ref. \\
\hline $\mathrm{B} 1516+02 \mathrm{~B}$ & 7.95 & 164.6 & $2.08 \pm 0.19$ & $0.13^{*}$ & 1 \\
$\mathrm{~B} 1855+09$ & 5.36 & 295.2 & $1.6 \pm 0.2$ & $0.258_{-0.03}^{+0.02}$ & 2 \\
$\mathrm{~B} 1957+20$ & 1.61 & 9.17 & $2.40 \pm 0.12$ & $0.035_{-0.02}^{+0.01}$ & 3 \\
$\mathrm{~J} 0024-7204 \mathrm{H}$ & 3.21 & 60.9 & $1.41_{-0.08}^{+0.04}$ & $0.18_{-0.02}^{+0.09}$ & 4 \\
$\mathrm{~J} 0437-4715$ & 5.76 & 137.8 & $1.76 \pm 0.20$ & $0.254 \pm 0.018$ & 5 \\
$\mathrm{~J} 0751+1807$ & 3.48 & 6.3 & $1.26 \pm 0.14$ & $0.19 \pm 0.03$ & 6 \\
$\mathrm{~J} 1012+5307$ & 5.26 & 14.5 & $1.68 \pm 0.22$ & $0.16 \pm 0.02$ & 7 \\
$\mathrm{~J} 1614-2230$ & 3.15 & 208.8 & $1.97 \pm 0.04$ & $0.4^{*}$ & 8 \\
$\mathrm{~J} 1713+0747$ & 4.57 & 1627.8 & $1.53_{-0.06}^{+0.08}$ & $0.33 \pm 0.04$ & 9 \\
$\mathrm{~J} 1738+0333$ & 5.85 & 8.52 & $1.55 \pm 0.55$ & $0.2 \pm 0.05$ & 10 \\
$\mathrm{~J} 1740-5340 \mathrm{~A}$ & 3.65 & 32.5 & $1.53 \pm 0.19$ & $0.22^{*}$ & 11 \\
$\mathrm{~J} 1903+0327$ & 2.15 & 2284.2 & $1.74 \pm 0.04$ & $1.05 \pm 0.02$ & 12 \\
$\mathrm{~J} 1909-3744$ & 2.95 & 36.7 & $1.47_{-0.02}^{+0.03}$ & $0.2038 \pm 0.0022$ & 13 \\
$\mathrm{~J} 1911-5958 \mathrm{~A}$ & 3.27 & 20.6 & $1.4_{-0.16}^{+0.16}$ & $0.18 \pm 0.02$ & 14 \\
$\mathrm{~J} 1748-2446 \mathrm{I}$ & 9.57 & 31.87 & $1.87_{-0.07}^{+0.32}$ & $0.24^{*}$ & 15 \\
$\mathrm{~J} 1045-4509$ & 7.47 & 97.92 & $<1.48$ & 0.13 & 16 \\
$\mathrm{~J} 1804-2718$ & 9.34 & 266.4 & $<1.73$ & 0.2 & 16 \\
$\mathrm{~J} 2019+2425$ & 3.93 & 1836 & $<1.51$ & $0.32-0.35$ & 17 \\
$\mathrm{~J} 1023+0038$ & 1.69 & 4.75 & $1.0-3.0$ & $0.14-0.42$ & 18 \\
$\mathrm{~J} 1738+0333$ & 5.85 & 8.50 & $1.6 \pm 0.2$ & 0.2 & 19 \\
$\mathrm{~J} 0024-7204 \mathrm{I}$ & 3.49 & 5.52 & 1.44 & 0.15 & 20 \\
$\mathrm{~J} 1518+0204 \mathrm{~B}$ & 7.95 & 164.64 & $2.08 \pm 0.19$ & $>0.13$ & 21 \\
$\mathrm{~J} 1824-2452 \mathrm{C}$ & 4.16 & 193.87 & $<1.367$ & $>0.26$ & 21 \\
$\mathrm{~J} 0514-4002 \mathrm{~A}$ & 4.99 & 450.96 & $<1.52$ & $>0.96$ & 22 \\
$\mathrm{~J} 1748-2021 \mathrm{I}$ & 9.57 & 31.87 & $1.3 \pm 0.02$ & 0.24 & 15 \\
\hline
\end{tabular}

Notes: *: Medium companion mass from ATNF pulsar database. 1.Freire et al 2008a, 2.Splaver 2004, 3.van Kerkwijk et al. 2011, 4.Freire et al. 2003, Manchester et al. 1991, 5.Verbiest et al. 2008, 6.Nice et al. 2008, 7.Lange et al. 2001, 8.Demorest et al. 2010, 9.Splaver et al. 2005, 10.Freire et al. 2008b, 11.Kaluzny et al. 2003, 12.Champion et al. 2008, 13.Jacoby et al. 2005, Hotan et al. 2006, 14.Bassa et al. 2006, 15.Ransom et al. 2005, 16.Thorsett \& Chakrabarty 1999, 17.Nice, Splaver, \& Stairs 2001, 18.Archibald et al. 2009, 19.Jacoby 2004, 20.Manchester et al. 1991, 21.Zhang et al. 2011, 22.Freire et al. 2007

King et al. (1996), long-period LMXBs are likely to be transient; for LMXBs with $P_{\text {orb }} \lesssim 2$ days, most neutron star systems will be persistent X-ray sources unless they have extreme mass ratios (i. e., $q<0.18 P_{\text {orb hr }}$ ). Consider the calculated results in Figs. 1-3, we conclude that mass ejection is more likely to occur in wide systems with light secondary (or in the late the evolution of long-period LMXBs) via disruption of the accretion disk inside the Roche lobe of the primary.

\section{SUMMARY AND DISCUSSION}

We can summarize the above results for the conditions of pulsar-driven mass ejection as follows.

(1) Pulsar-driven mass ejection is likely to occur in wide LMXBs (in case 1 evolution), in which the thermal instability in the accretion disk can result in large variations in the accretion rate.

(2) LMXBs in case 2 evolution may barely be subject to mass ejection.

(3) If there is mass ejection, it may be caused by the disruption of the accretion disk leading to outflow through the $L_{1}$ point. When the secondary is of extremely low mass, outflow from the $L_{2}$ point is also possible.

An important factor that was neglected in the above sections is irradiation in LMXBs caused by accretion-generated X-rays. It was realized that irradiation of the donor star or the accretion disk can not only change the optical appearance of LMXBs (King \& Ritter 1999), but also their outburst properties (van Paradijs 1996), and possibly the long-term evolution of the donor (Podsiadlowski 1991). Especially irradiation of the donor star can destabilize the mass transfer, and lead to mass transfer cycles (Hameury et al. 1993), which differs drastically from the evolution we considered above: mass transfer is spasmodic with phases of high mass transfer driven by the thermal expansion of the convective envelope 
of the irradiated donor alternating with phases with low or no mass transfer, during which the donor readjusts towards thermal equilibrium of the un-irradiated star (Büning \& Ritter 2004). As pointed out by Ritter (2008), the effect of irradiation may be important in compact rather long-period LMXBs, since in the later the irradiation resulting from accretion is intermittent due to disk instability. However, even in the former systems, the details of how irradiation of the donor influences the mass transfer process are very complicated, and have not been included in the calculations of the secular evolutions of I/LMXBs in a self-consistent way (Pfahl et al. 2003). It is then difficult to estimate the efficiency of mass ejection due to irradiation. On one hand, during the short "high" state the mass transfer rate is enhanced and larger than the secular one, leading to shorter $p_{\text {eq }}$, which is in favor of mass ejection (In Fig. 3, if the mass transfer rate is increased by a factor of 100 , both $p_{\mathrm{eq}}<p_{\mathrm{cr}}$ and $p_{\mathrm{eq}}<p_{\mathrm{cr}}^{\prime}$ will be satisfied). On the other hand, during the long "low" state, the mass transfer rate becomes much lower than the secular one, so that less mass can be blown off. This is in contrast with the limit cycles due to disk instability, in which the accretion rate varies by a large factor but the mass transfer rate remains nearly unchanged.

The current masses of MSPs may reveal possible evidence of mass accretion and ejection during the previous LMXB evolution. In Table 1 we list the parameters of binary MSPs with measured masse 2 . In general, long-period ( $P_{\text {orb }} \gtrsim 20-30$ days) MSPs have masses around $1.4 M_{\odot}$, suggesting that large amount of the transferred mass was lost. In narrow systems ( $P_{\text {orb }}<2-3$ days) the pulsar masses distribute from $\sim 1.3 M_{\odot}$ to $\sim 2.4 M_{\odot}$, indicating that both efficient mass accretion and mass ejection are possible, depending on the properties of the individual sources. However, a large fraction of them also have masses not far from 1.4 $M_{\odot}$. We speculate that irradiation-driven mass transfer cycles may help drive off the transferred mass from their companions in these systems.

Besides the standard recycling scenario, another possible way for mass ejection in short-period LMXBs is companion exchange. This should happen only in the environment with high stellar density like globular clusters. The incidence of black widow pulsars in globular clusters is known to be far higher than in the field. This leads King et al. (2003) to suggest that the MSPs in dead wide binaries with white dwarf companions have exchanged them for normal stars. Encounters and tides bring these new companions into tight orbits. Due to intense accretion during the first mass transfer phase, the neutron star's spin period may satisfy the condition $p_{\text {eq }} \leq p_{\text {cr }}$ or $p_{\text {cr }}^{\prime}$ with current mass transfer rate, resulting in mass ejection from the binary.

Finally, the condition for mass ejection may be satisfied if the accreting star in LMXBs is a strange star rather a neutron star. In the former case the spin periods could be submilliseconds (e.g., Frieman \& Olinto 1989; Gourgoulhon et al. 1999; Possenti et al. 1999), and less than the critical periods given by Eqs. (8) and (14). These exotic objects might be formed by accretion-induced collapse of white dwarfs in binary systems (Du et al. 2009).

Acknowledgements This work was supported by the Natural Science Foundation of China under grant number 10873008 and the Ministry of Science and the National Basic Research Program of China (973 Program 2009CB824800).

\section{Appendix A: CALCULATION OF THE DISK PRESSURE}

In the model of magnetic dominant disk Begelman and Pringle argued that the amplified toroidal magnetic field pressure is $\sim \rho c_{g} v_{k}$. They calculated the density inside the disc is

$$
\rho=\frac{\Sigma}{2 H} \sim \frac{2}{3} \frac{c^{2}}{G M \kappa}\left(\frac{c}{c_{g}}\right)^{3 / 2} \frac{\dot{M}}{\dot{M}_{E d d}} \alpha^{-1}\left(\frac{R}{R_{g}}\right)^{-9 / 4}
$$

here $\kappa$ is the opacity, $\dot{M}_{E d d}=4 \pi G M / \kappa c$ is the Eddington accretion rate and $R_{g}=G M / c^{2}$. In this model the viscosity and scale height of the disc is given by $\nu=\alpha H v_{A}$ and $H / R \sim\left(c_{g} / v_{k}\right)^{1 / 2}$ instead

\footnotetext{
2 PSR J1903+0327 is a peculiar MSP with a main-sequence companion star, and is not considered here.
} 
of $\nu=\alpha H c_{g}$ and $H / R=\left(c_{g}^{2}+c_{r}^{2}\right)^{1 / 2} / v_{k}$ in SS model. After these replacement then use the equation of angular momentum

$$
\nu \Sigma=\frac{\dot{M}}{3 \pi}\left[1-\left(\frac{R_{*}}{R}\right)^{1 / 2}\right]
$$

and energy

$$
\frac{4 \sigma}{3 \tau} T_{c}^{4}=\frac{3 G M \dot{M}}{8 \pi R^{3}}\left[1-\left(\frac{R_{*}}{R}\right)^{1 / 2}\right]
$$

we can calculate the disk pressure is

$$
P_{\text {disk }}=\alpha^{-17 / 18} \frac{(G M)^{61 / 36}}{6 \pi c^{2}}\left(\frac{3 \kappa}{32 \pi^{2} \sigma}\right)^{-1 / 18} \frac{\dot{M}^{8 / 9}}{R_{n}}\left(\frac{k}{\mu m_{p}}\right)^{-2 / 9} R^{-91 / 36}\left[1-\left(\frac{R_{*}}{R}\right)^{1 / 2}\right]^{-1 / 9} \text {. }
$$

\section{References}

Archibald A. M., Stairs I. H., Ransom S. M. et al., 2009, Science, 324, 1411

Balbus S. A., Hawley J. F., 1998, Rev. Mod. Phys., 70, 1

Bassa, C.G., van Kerkwijk, M.H., Koester, D. \& Verbunt, F. 2006, A\&A, 456, 295

Begelman, M. C. \& Pringle, J. E., 2007, MNRAS, 375, 1070

Bhattacharya, D., van den Heuvel, E. P. J., 1991, Phys. Rep., 203, 1

Büning, A, \& Ritter, H., 2004, A\&A 423, 281

Burderi, L., D’Antona, F. \& Burgay, M. 2002, ApJ, 574, 325

Burderi, L., Possenti, A., Colpi, M., Di Salvo,T., \& D‘Amico, N. 1999, ApJ, 519, 285

Burderi, L., Possenti, A., D’Antona, F. , et al. 2001, ApJ, 560, L71

Champion, D.J., Ransom, S.M., Lazarus, P. et al., 2008, Science, 320, 1309

D’Amico, N., Possenti, A., Manchester, R. N., Sarkissian, J., Lyne, A. G., Camilo, F., 2001, ApJ, 561, L89

Davies, M.B. \& Hansen, B.M., 1998, MNRAS, 301, 15

Demorest P. B., Pennucci T., Ransom, S. M., et al. 2010, Nature, 467, 1081

Du, Y.-J., Xu, R.-X., Qiao, G.-J., \& Han, J.-L. 2009, MNRAS, 399, 1587

Ferraro, F. R., Possenti, A., D’Amico, N., Sabbi, E. 2001, ApJ, 561, L93

Frank, J., King, A. R., \& Raine, D. 2002, Accretion power in astrophysics, Cambridge University Press

Freire, P.C.C., Wolszcan, A., van den Berg,M. et al, 2008a, ApJ, 679, 1433

Freire P. C., Ransom S. M. \& Gupta Y. 2007, ApJ, 662, 1177

Freire, P.C.C., Camilo, F., Kramer, M., et al. 2003 MNRAS, 340, 1359

Freire, P.C.C., Jacoby, B.A., \& Bailes,M., 2008b, AIP Conference Proceedings 983, 488; arXiv:0711.1880

Frieman, J. A., \& Olinto, A. V. 1989, Nature, 341, 633

Fruchter, A. S., Stinebring, D. R., Taylor, J. H. 1988, Nature, 312, 255

Ghosh, P., \& Lamb, F. K. 1979, ApJ, 234, 296

Gourgoulhon, E., Haensel, P., Livine, R., Paluch, E., Bonazzola, S., \& Marck, J.-A. 1999, A\&A, 349, 851

Hakala P. J., Muhli P., Dubus G., 1999, MNRAS, 306, 701

Hameury J. M., King A. R., Lasota J.P. et al. 1993, A\&A, 277, 81

Hotan, A.W., Bailes, M. \& Ord, S.M., 2006, MNRAS, 369, 1502

Illarionov, A. F. \& Sunyaev, R. A. 1975, A\&A, 39, 185

Jacoby B. A. 2004, PhD thesis, Calfornia Institute of Technology

Jacoby, B. A., Hotan, A., Bailes, M., Ord, S., \& Kulkarni, S. R. 2005, ApJ, 629, L113

Kaluzny, J., Rucinski, S. M., \& Thompson, I. B. 2003, AJ, 125, 1546

Kiziltan, B., Kottas, A., \& Thorsett, S. E. 2010, arXiv:1011.4291

King, A. R. 1988, QJRAS, 29, 1

King, A.R., Davies, M.B. \& Beer, M.E., 2003, MNRAS, 345, 678

King, A. R., Kolb, U., Burderi, L., 1996, ApJ, 464, L127 
King, A. R. \& Ritter, H. 1999, MNRAS, 309, 253

Landau, L., \& Lifschitz, E. 1958, Classical Theory of Fields (Elmsford: Pergamon)

Lange, Ch., Camilo, F., Wex, N., et al. 2001 MNRAS, 326, 274 (2001)

Lasota, J.-P. 2001, NewAR, 45, 449

Li, S.-L., Miller, N. Lin, D.N.C., Fortney, et al. 2010, Nature, 463, 1054

Manchester R. N., Lyne A. G., Robinson C., et al. 1991, Nature, 352, 219

Nice, D. J., Splaver, E. M. \& Stairs, I. H. 2001, ApJ, 549, 516

Nice, D. J., Splaver, E. M., Stairs, I. H., et al. 2005, ApJ, 634, 1242

Nice, D.J., Stairs, I.H. \& Kasian, L.E., 2008, AIP Conference Proceedings 983, 453

Pfahl, E. D., Rappaport, S. \& Podsiadlowski, P., 2003, ApJ, 5971036

Phillips, S. N. \& Podsiadlowski, P. 2002, MNRAS, 337, 431

Podsiadlowski, P. 1991, Nature, 350, 136

Podsiadlowski, P., Rappaport, S. \& Pfahl, E. D. 2002 ApJ, 565, 1107

Possenti, A., Colpi, M., Geppert, U., Burderi, L., \& D’Amico, N. 1999, ApJS, 125, 463

Ransom, S. M., Hessels, J.W.T., Stairs, I.H., et al. 2005, Science, 307, 892

Ritter, H. 2008, NewAR, 51, 869

Ruderman, M., Shaham, J. \& Tavani, M. 1989, ApJ, 336, 507

Shafter A.W., Misselt K. A., 2006, ApJ, 644, 1104

Shaham, J. \& Tavani, M. 1991, ApJ, 377, 588

Shakura, N. I. \& Sunyaev, R. A., 1973, A\&A, 24, 337

Splaver, E. M., 2004, PhD Thesis, Princeton University

Splaver, E. M., Nice, D. J., Stairs, I. H., Lommen, A. N., \& Backer, D. C. 2005, ApJ, 620, 405

Tauris, T. M., Langer, N., \& Kramer, M. 2011, MNRAS, in press (arXiv:1103.4996)

Tauris, T. M. \& Savonije, G. J. 1999, A\&A, 350, 928

Thorsett, S. E. \& Chakrabarty, D. 1999, ApJ, 512, 288

van den Heuvel, E. P. J. \& van Paradijs, J. 1988, Nature, 334, 227

van Kerkwijk, M. H., Breton, R., \& Kulkarni, S. R., 2011, ApJ, 728, 95

van Paradijs, J., 1996, ApJ 464, L139

Verbiest, J. P. W., Bailes, M., van Straten, W. et al., 2008, ApJ, 679, 675

Verbunt, F., \& Zwaan, C. 1981, A\&A, 100, L7

Webbink, R. F., Rappaport, S., \& Savonije, G. J. 1983, ApJ, 270, 678

Zhang C. M., Wang J., Zhao Y. H. et al. 2011, A\&A, 527, 83 